CARTA AL EDITOR

\title{
Base biológica y estadificación del síndrome de Cotarrd
}

\author{
H. S. Duggal, K. Jagadheesan y S. Haque Nizamie \\ Instituto Central de Psiquiatría, Kanke, Ranchi - 834 006, Jharkhand, India
}

\begin{abstract}
Recientemente, ha habido un resurgimiento del interés en el síndrome de Cotard $[1,2,11]$. Además de explorar su base biológica [5-7, 9], se ha hecho un intento de estadificar este síndrome [11]. Se describe un caso de síndrome de Cotard manteniendo en segundo plano las tendencias recientes en su fundamentación neurobiológica y estadificación.

Un hombre de 32 años, que tenía antecedentes de trastorno bipolar en una tía materna, acudió a consulta cuando llevaba enfermo unos 14 meses. Para empezar, tuvo sensaciones extrañas de comienzo repentino en el interior de la cabeza y el abdomen. Simultáneamente, se volvió triste y comenzó a dormir mal y a perder el apetito. En las 3 semanas siguientes, desarrolló una convicción firme de que no tenía cerebro ni corazón. En esa época, después de la visualización de una escena alucinada que le representaba teniendo relaciones sexuales con su esposa, se puso ansioso y consultó con un médico local. Las pastillas sedantes prescritas no le proporcionaron ninguna mejoría; más bien sus síntomas depresivos empeoraron y de ahí que nos consultara.
\end{abstract}

La exploración física era normal y también el análisis de sangre, el ECG y el TAC cerebral. El EEG mostró anomalías de fondo no específicas. Después de obtener puntuaciones altas en el Test Gestáltico Visomotor de Bender, se llevó a cabo la Batería de Strub y Black [10], que reveló disfunción del lóbulo frontal y parietal (apraxia constructiva). La exploración psiquiátrica reveló afecto ansioso e irritable, ideas delirantes nihilistas prominentes de que no tenía cerebro y corazón e ideas delirantes de persecución. Además de sus pensamientos depresivos, también relató retros- pectivamente un único episodio de alucinaciones visuales.

El tratamiento se inició con $25 \mathrm{mg} / \mathrm{d}$ de amitriptilina y $3 \mathrm{mg} / \mathrm{d}$ de flupentixol. A los 2 días del inicio de estos fármacos, desarrolló síntomas maníacos; por tanto, se interrumpió la amitriptilina y se inició valproato. Sorprendentemente, sus ideas delirantes nihilistas desaparecieron en esos 2 días, si bien las persecutorias persistieron. Después de la resolución de la manía con valproato sódico $(600-800 \mathrm{mg} / \mathrm{d})$ y flupentixol $(3 \mathrm{mg} / \mathrm{d})$ fue dado de alta del hospital.

Además de la negación de la existencia de órganos vitales, un tipo de idea delirante nihilista, el paciente también tenía otros rasgos que acompañan al síndrome de Cotard, a saber, sensaciones corporales, alucinaciones visuales e ideación suicida [3].

La negación de la existencia de partes del cuerpo normales es un signo de disfunción del lóbulo parietal, que se produce también en las lesiones del lóbulo frontal dorsolateral, el giro cingulado, el tálamo y la neocorteza (caudado y putamen) [4]. Notablemente, la negación de órganos es también el rasgo esencial del síndrome de Cotard y las anomalías observadas frontales y frontoparietales [5-7, 9] indican la posible implicación de estas regiones en el comienzo de la idea delirante nihilista.

De modo similar a otro informe [11], el perfil longitudinal de la enfermedad de nuestro paciente se puede dividir también en: estadio de germinación (depresión, cenestopatía e hipocondría), estado de florecimiento (desarrollo completo de los síntomas de estadio de Cotard) y estadio crónico (sistematización y cambio a paranoia con retroceso de la depresión). El estadio cró- 
nico en este paciente se cortó en seco por la aparición de síntomas maníacos. Ya que las crisis, artificiales o naturales [8], alivian este síndrome, se puede exponer un papel similar para el cambio maníaco advertido en este caso considerando que la activación propagada (kindling) es una hipótesis aceptada para la bipolaridad.

\section{BIBLIOGRAFÍA}

1 Berrios GE; Luque R. Cotard's delusion or syndrome?: a conceptual history. Compr Psychiatry 1995; 36: 218-23.

2 Berrios GE, Luque R. Cotard's syndrome: analysis of 100 cases. Acta Psychiatr Scand 1995 i 91: 185-8.

3 Cotard J. Nihilistic delusions. In: Hirsch SR, Shepherd $\mathrm{M}$, Eds. Themes and variations in European psychiatry: an anthology. Bristol: John Wright \& Sons; 1974. p. 353-73.

4 Cummings JL. Clinical neuropsychiatry. Orlando: Grune \& Stratton, Inc; 1985. p. 48-56.
5 Enoch MD, Trethowan WH. Uncommon psychiatric syndromes, 2nd Ed. Bristol: John Wright and Sons; 1979.

6 Joseph AB, O'Leary $\mathrm{DH}$. Brain atrophy and interhemispheric enlargement in Cotard's syndrome. J Clin Psychiatry 1986; 47: 518-20.

7 Joseph AB. Cotard's syndrome in a patient with coexistent Capgras' syndrome, syndrome of subjective doubles, and palinopsia. J Clin Psychiatry 1986; 47: 605-6.

8 Malone $\mathrm{K}$, Malone JP. Remarkable resolution of an uncommon psychosyndrome: epilepsy-induced remission of Cotard's syndrome. Ir J Psychol Med 1992; 9: 53-4.

9 Petracca G, Migliorelli R, Vazquez S, Starkstein SE. SPECT findings before and after ECT in a patient with major depression and Cotard's syndrome. J Neuropsychiatry Clin Neurosci 1995; 7: 505-7.

10 Strub RL, Black FW. The mental status examination in neurology, 3rd ed. Philadelphia: F.A. Davis Company; 1993.

11 Yamada K, Katsuragi S, Fujii I. A case study of Cotard's syndrome: stages and diagnosis. Acta Psychiatr Scand 1999; 100: 396-9. 